\title{
Characterization of superconducting RF breakdown by two-mode excitation
}

\author{
G. Eremeev and A.D. Palczewski \\ Thomas Jefferson National Accelerator Facility, Newport News, VA 23606, U.S.A.
}

(Dated: October 5, 2018)

\begin{abstract}
We show that thermal and magnetic contributions to the breakdown of superconductivity in radiofrequency $(\mathrm{RF})$ fields can be separated by applying two RF modes simultaneously to a superconducting surface. We develop a simple model that illustrates how mode-mixing RF data can be related to properties of the superconductor. Within our model the data can be described by a single parameter, which can be derived either from RF or thermometry data. Our RF and thermometry data are in good agreement with the model. We propose to use mode-mixing technique to decouple thermal and magnetic effects on RF breakdown of superconductors.
\end{abstract}

\section{INTRODUCTION}

With the advances in superconducting radiofrequency (SRF) cavity fabrication and preparation over the last four decades, the peak surface magnetic field in superconducting niobium cavities approaches the critical field of niobium [1, 2]. The best SRF cavities lose their superconductivity at about $B_{\text {peak }}=180-200 \mathrm{mT}$, which is close to the thermodynamic critical field of niobium 3 . Theoretical calculations suggest the scaling of the superheating field, which is believed to be the limitation of superconductivity in RF fields, to the thermodynamic critical field [4, [5], [6]. Experimental data near the transition temperature supports the superheating critical field as the limitation in RF fields [7, but in experiments at low temperatures SRF cavities are often limited to fields lower than the expected superheating field, possibly due to local imperfections. How the cavity quench field relates to the material critical field is not evident, because $\mathrm{RF}$ dissipation increases the temperature of RF surface and magnetic and thermal effects on the superconducting $\mathrm{RF}$ breakdown become entangled.

We show that thermal and magnetic effect can be decoupled by exciting two RF modes. The idea was exploited in 1980 by D. Proch et al., guided by the intuitive understanding that magnetic breakdown and thermal breakdown will behave differently in time-varying fields [8]: in a multicell SRF cavity the surface field distribution in each cell is the same among all resonances of a fundamental mode passband, but relative field strengths between different cells vary from mode to mode. If two pass-band modes in a cell are excited at the same time, their field vectors are collinear, but they do not add up coherently because of different resonant frequencies. The incoherent summation leads to beating in the amplitude of the resulting wave, where the maximum field is equal to the sum of field amplitudes, and the time averaged dot product of two fields is zero. The thermal breakdown will be determined by the sum of field amplitudes squared, while magnetic breakdown is the consequence of maximum field amplitude. D. Proch et al. studied $\pi$ and

\footnotetext{
* grigory@jlab.org
}

$\pi / 2$ excitation in several 2-cell cavities. By bringing cavities into repetitive quench with varying field strengths ratio of two modes, quench dependence on field amplitude ratio was measured. The conclusion was that "the data unambiguously supports the thermal model."

Our data deviates from the quadratic law that we expected for thermal instability. We explain the data by magnetic breakdown on surface features with thermally suppressed critical field, and model how thermal and magnetic properties of the material result in the observed behavior. The experimental technique can be applied to characterize SRF niobium cavities as well as to characterize the RF breakdown of superconductivity in other superconducting devices.

\section{RF BREAKDOWN BY TWO-MODE EXCITATION}

Superconducting properties of an SRF cavity are typically described by the curve showing the quality factor as a function of the RF field. The best superconducting cavities reach fields up to $200 \mathrm{mT}$ with a decrease in the quality factor, so-called medium field Q-slope. Temperature mapping shows that temperature increase of the sensors' temperature over the superfluid helium bath temperature is roughly quadratic with the field in this regime 9 . The temperature increase of the sensor's temperature corresponds to the temperature increase on the RF surface of the cavity, so we will use quadratic approximation of the RF surface temperature:

$$
T_{\mathrm{RF}} \cong T_{0}+A \cdot H_{\mathrm{RF}}^{2},
$$

where $T_{0}$ is the liquid helium bath temperature, and $A$ is the coefficient of proportionality. To understand how coefficient A depends on niobium wall properties, let us consider a simple model.

Following 10, let us consider a one-dimensional geometry, where a slab of niobium with thickness $d=3 \mathrm{~mm}$ is cooled by a liquid helium bath at $2 \mathrm{~K}$ on one side, and on the other side a time-varying RF field $\vec{H}_{\mathrm{RF}}$ is applied in vacuum. The characteristic propagation time for a thermal wave in this geometry is $\tau_{T} \approx d^{2} C_{v} / \kappa[11$. Niobium volumetric heat capacity $C_{v}$ is $\approx 10^{3} \mathrm{~J} /\left(\mathrm{K} \cdot \mathrm{m}^{3}\right)[12$ and 
the thermal conductivity $\kappa$ is $\approx 10 \mathrm{~W} /(\mathrm{K} \cdot m)$ at $2 \mathrm{~K}$, so $\tau_{T} \approx 1$ msec. This time is much smaller than RF field amplitude modulation times $Q_{L} / \omega \cong Q_{0} / \omega \approx 1$ sec for a critically coupled cavity with a typical intrinsic quality factor $Q_{0} \cong 10^{10}$, but it is much larger than $\mathrm{RF}$ period $1 / \mathrm{f} \cong 1 \mathrm{nsec}$, so the heat flow equation can be reduced to steady state simultaneous equations:

$$
\begin{aligned}
R_{s}\left(T_{\mathrm{RF}}\right) \overline{\vec{H}_{\mathrm{RF}}^{2}} d & =\int_{T_{s}}^{T_{\mathrm{RF}}} \kappa(T) d T \\
R_{s}\left(T_{\mathrm{RF}}\right) \overline{\vec{H}_{\mathrm{RF}}^{2}} & =H_{\text {Kapitza }}\left(T_{s}, T_{0}\right) \cdot\left(T_{s}-T_{0}\right),
\end{aligned}
$$

where $R_{s}\left(T_{\mathrm{RF}}\right)$ is the surface resistance, $\kappa(T)$ is the thermal conductivity of niobium, $H_{\text {Kapitza }}\left(T_{s}, T_{0}\right)$ is the Kapitza conductance, $\overrightarrow{\vec{H}}_{\mathrm{RF}}^{2}$ is the time-averaged RF field squared, $T_{0}$ is the helium bath temperature, $T_{\mathrm{s}}$ is the temperature of the niobium surface on the helium side, and $T_{\mathrm{RF}}$ is the temperature of RF side. The pre-heating temperature rise before a quench measured on the surface of a superconducting RF cavity is usually on the order of $0.1 \mathrm{~K}\left[10\right.$, 13], so assuming $\kappa(T) \cong \kappa\left(T_{0}\right)=\kappa$ and $H_{\text {Kapitza }}\left(T_{\mathrm{s}}, T_{0}\right) \cong H_{\text {Kapitza }}\left(T_{0}, T_{0}\right)=H_{\text {Kapitza }}$ the temperature on the RF surface can be expressed:

$$
T_{\mathrm{RF}} \cong T_{0}+C \cdot R_{\mathrm{s}} \overline{\vec{H}_{\mathrm{RF}}^{2}}
$$

where $C$ is $\left(\frac{d}{\kappa}+\frac{1}{H_{\text {Kapitza }}}\right)$.

When two modes are excited, the resulting field is the vector sum of two fields, $\vec{H}_{\mathrm{RF}}=\vec{H}_{1} e^{i \omega_{1} t}+\vec{H}_{2} e^{i \omega_{2} t}$, where $\vec{H}_{1}, \vec{H}_{2}$ field amplitudes are parallel for $\mathrm{TM}_{010}$ passband modes. Since the thermal diffusion time is much larger than $2 \pi / \omega_{1}, 2 \pi / \omega_{2}$, and $2 \pi /\left(\left|\omega_{1}-\omega_{2}\right| \approx 1 \mu \mathrm{sec}\right.$, averaging the RF field over the thermal diffusion time results in $\overrightarrow{\vec{H}_{\mathrm{RF}}^{2}}=\frac{1}{2}\left(H_{1}^{2}+H_{2}^{2}\right)$. Hence, when two fields are applied:

$$
T_{\mathrm{RF}} \cong T_{0}+C \frac{1}{2} R_{s}\left(H_{1}^{2}+H_{2}^{2}\right)
$$

The result illustrates that the characteristic times for a typical SRF cavity are such that the total dissipation on the RF surface can be expressed as the sum of losses. The result supports the intuitive speculation that the power losses of two passband modes in an SRF cavity add up independently.

For magnetic breakdown we expect niobium to go from superconducting- to normal-conducting state, when the applied RF magnetic field exceeds the RF critical field on the surface. We will assume that the magnetic breakdown occurs when the amplitude of magnetic field exceeds the RF critical field, that is in the case of mixed modes we assume that the breakdown occurs when the sum of RF field amplitudes exceeds the RF critical field, i.e., $H_{1}+H_{2}=H_{\text {crit }}^{\mathrm{RF}}(T)$. The temperature dependence of $H_{\text {crit }}^{\mathrm{RF}}(T)$ is still under discussion. The measurements of T. Hays et al. 14] as well as more recent ones by N. Valles et al. 15 are fitted with the accepted approximation for the thermodynamic critical field, while earlier measurements of T. Yogi[7] show a divergence of $\mathrm{H}_{c}^{\mathrm{RF}} / \mathrm{H}_{c}$ near $\mathrm{T}_{c}$. Recent theoretical calculations [4, [5, [6] show non-trivial dependence of $\mathrm{H}_{c}^{\mathrm{RF}} / \mathrm{H}_{c}$ within different approximations. Since there is no universally accepted RF critical field approximation for niobium at $2 \mathrm{~K}$ with the Ginsburg-Landau parameter $\cong 1$, we will use the textbook formula for the temperature dependence of the thermodynamic critical field [16] to model the temperature dependence of RF critical field:

$$
H_{c}\left(T_{R F}\right)=H_{c}\left(1-\left(\frac{T_{R F}}{T_{C}}\right)^{2}\right),
$$

where $H_{c}$ is $H_{c}(0)$, the critical field at zero temperature, $T_{c}$ is the critical temperature, and $T_{R F}$ is the temperature of the RF surface. When two RF fields are applied, the sum of the field amplitudes of two modes is equal to the critical magnetic field:

$$
H_{1}+H_{2}=H_{c}\left(1-\left(\frac{T_{0}+C \frac{1}{2} R_{s}\left(H_{1}^{2}+H_{2}^{2}\right)}{T_{C}}\right)^{2}\right),
$$

where $T_{c}$ and $H_{c}$ are the critical temperature and the magnetic field, and we used (3) for RF surface temperature. When only one field is applied:

$$
H_{\max }=H_{c}\left(1-\left(\frac{T_{0}+C \frac{1}{2} R_{s} H_{\max }^{2}}{T_{C}}\right)^{2}\right),
$$

where $H_{\max }$ is the breakdown field amplitude. Equations (6) and (7) can be reduced to:

$$
\begin{aligned}
& \left(\frac{H_{1}}{H_{\max }}+\frac{H_{2}}{H_{\max }}\right)+ \\
& +\frac{R_{s} H_{c} T_{0} H_{\max } C}{T_{c}^{2}} \times\left(\left(\frac{H_{1}}{H_{\max }}\right)^{2}+\left(\frac{H_{2}}{H_{\max }}\right)^{2}\right)+ \\
& +\frac{R_{s}^{2} H_{c} H_{\max }^{3} C^{2}}{4 T_{c}^{2}} \times\left(\left(\frac{H_{1}}{H_{\max }}\right)^{2}+\left(\frac{H_{2}}{H_{\max }}\right)^{2}\right)^{2}= \\
& =1+\frac{R_{s} H_{c} T_{0} H_{\max } C}{T_{c}^{2}}+\frac{R_{s}^{2} H_{c} H_{\max }^{3} C^{2}}{4 T_{c}^{2}}
\end{aligned}
$$

If we define $1 / \alpha=1+\frac{R_{s} H_{c} T_{0} H_{\max } C}{T_{c}^{2}}+\frac{R_{s}^{2} H_{c} H_{\max }^{3} C^{2}}{4 T_{c}^{2}}$ and normalize the fields $H_{1}$ and $H_{2}$ to $\mathrm{H}_{\max }$, the resulting equation can be written in terms of $\alpha, T_{0}, T_{c}$ and normalized fields $\tilde{H}_{1}$ and $\tilde{H}_{2}$ as:

$$
\begin{aligned}
& \alpha\left(\tilde{H}_{1}+\tilde{H}_{2}\right)+ \\
& +2 \frac{T_{0}^{2}}{T_{c}^{2}-T_{0}^{2}}\left(\sqrt{\frac{T_{c}^{2}}{T_{0}^{2}}(1-\alpha)+\alpha}-1\right)\left(\tilde{H}_{1}^{2}+\tilde{H}_{2}^{2}\right)+ \\
& +\frac{T_{0}^{2}}{T_{c}^{2}-T_{0}^{2}}\left(\sqrt{\frac{T_{c}^{2}}{T_{0}^{2}}(1-\alpha)+\alpha}-1\right)^{2}\left(\tilde{H}_{1}^{2}+\tilde{H}_{2}^{2}\right)^{2}=1
\end{aligned}
$$


To understand intuitively the physical meaning of $\alpha$, we will assume that $\alpha$ is close to 1 , that is $\alpha=1-\delta$, where $\delta<<1$. Expanding equation (9) and dropping quadratic and higher order terms in $\delta$, we obtain the following expression for mode-mixing:

$$
\alpha\left(\tilde{H}_{1}+\tilde{H}_{2}\right)+(1-\alpha)\left(\tilde{H}_{1}^{2}+\tilde{H}_{2}^{2}\right)=1
$$

From this equation we can see that $\alpha=1$ means a purely magnetic quench. As $\alpha$ departs from one, the thermal effects become more pronounced.

To understand the physical meaning of $\alpha$ quantitatively, we expand the equation (6) and, after combining with equation (8) and the definition of $\alpha$, the fitting parameter $\alpha$ can be re-written as:

$$
\alpha=\frac{H_{\max }}{H_{c}\left(1-\left(\frac{T_{0}}{T_{C}}\right)^{2}\right)}
$$

This expression illustrates the physical meaning of the fitting parameter $\alpha$. The fitting parameter quantifies how much the material critical field is suppressed due to RF surface temperature increase over the liquid helium bath temperature. In analogy with geometrical field enhancement factor, $\alpha$ can be called the thermal suppression factor.

Equation 10 clearly shows that pre-quench temperature rise varies with $\alpha$. In the case of $\alpha=0$, the prequench temperature rise is the same for all mixed field amplitudes; in the case of $\alpha=1$, the pre-quench temperature rise dependence on one of the fields is parabolic with $\tilde{H}_{\min }=0.5$ and $\Delta T_{\min } / \Delta T_{\max }=0.5$. Here $\Delta T_{\max }$ is the maximum pre-heating temperature rise during mode mixing, $\Delta T_{\min }$ is the minimum pre-heating temperature rise, and $\tilde{H}_{\text {min }}$ is the normalized field level at which the minimum temperature rise occurs.

\section{EXPERIMENTAL SETUP}

All studied cavities followed the current standard the International Linear Collider (ILC) cavity preparation procedure 17. A general overview of modern preparation techniques can be found in [18. Quality factor vs. field for $\pi$ and other $\mathrm{TM}_{010}$ pass-band cavity modes was measured using conventional phase-lock loop techniques; see e.g.,[19]. All RF tests were done in the liquid helium bath at $2 \mathrm{~K}$. Helium temperature was kept constant by cryosystem feedback and was monitored by a calibrated temperature sensor in the helium bath. For the pass-band measurements the transmitted power from the pick-up probe was used to calculate the RF field amplitude in the end cells; the RF fields for the other cells were calculated using the end cell amplitude 20 .

During measurements, second sound propagation in the liquid helium bath 21] and temperature mapping techniques 22 were used to identify quench locations, and to confirm that breakdown happens in the same location during the mode-mixing measurement. All the passband modes of a cavity are measured first and the highest field in each cell for each mode is calculated. Quench locations are determined with oscillating superleak transducers (OSTs) and the limiting cell for each pass-band mode is identified. After OSTs and thermometry data have been analyzed, two modes that are limited by the same defect are chosen for mode-mixing measurements.

Once the pass-band modes are chosen, we use two independent voltage-controlled oscillators (VCOs) with independent phase-lock loops (PLL) to drive modes independent of each other. Each VCO is tuned to one of the modes. The drive signals from VCOs are combined with a power combiner and fed into the high power RF amplifier. From the power amplifier through RF cables and the directional coupler, RF power from two sources is fed into the cavity via the input coupler of the cavity. Phase locking is accomplished by splitting transmitted power from the pick-up probe with power dividers and feeding the transmitted signal into the PLL of both VCOs. Part of the transmitted power is coupled into the input port of the spectrum analyzer. The spectrum analyzer is then used to measure field level of each mode during mode-mixing experiments. More details about setup, measurement technique, and calibration can be found elsewhere 23 .

Measured mode-mixing data is normalized to the quench field in each mode and presented on an x-y plot. Each data point on the $\mathrm{x}-\mathrm{y}$ plot corresponds to a single $\mathrm{RF}$ breakdown event during mode-mixing. The abscissa of the point is equal to one of the mixed fields normalized amplitudes; the ordinate of the point is equal to the other field normalized amplitude. As the result an $x-y$ dependence connecting points $(0,1)$ and $(1,0)$ is plotted. To fit the experimental data we used:

$$
\begin{aligned}
& \alpha\left(\tilde{H}_{1}+\tilde{H}_{2}\right)+ \\
& +2 \frac{T_{0}^{2}}{T_{c}^{2}-T_{0}^{2}}\left(\sqrt{\frac{T_{c}^{2}}{T_{0}^{2}}(1-\alpha)+\alpha}-1\right)\left(\tilde{H}_{1}^{2}+\tilde{H}_{2}^{2}\right)+ \\
& +\frac{T_{0}^{2}}{T_{c}^{2}-T_{0}^{2}}\left(\sqrt{\frac{T_{c}^{2}}{T_{0}^{2}}(1-\alpha)+\alpha}-1\right)^{2}\left(\tilde{H}_{1}^{2}+\tilde{H}_{2}^{2}\right)^{2}=1,
\end{aligned}
$$

where $\alpha$ is a fitting parameter and $\tilde{H}_{1}$ and $\tilde{H}_{2}$ are the field amplitudes of each mode normalized to the maximum field amplitude measured with single-mode excitation. The critical temperature $T_{c}$ is set to $9.25 \mathrm{~K}$, and the bath temperature $T_{0}$ is set to $2 \mathrm{~K}$.

Thermometry is commonly used in an SRF field to measure dissipation distribution on the cavity surface. The temperature measured by a temperature sensor on the outside surface of a cavity is proportional to the temperature on the inside surface. It is commonly accepted that for Allen-Bradley thermometry 24, which is widely used in SRF fields, the measured temperature is propor- 
tional to the power flux flowing though the sensor from the RF surface into the liquid helium bath:

$$
\Delta T \propto P^{\mathrm{RF}}=\overline{R_{s}\left(\vec{H}_{1}+\vec{H}_{2}\right)^{2}}=\frac{1}{2} R_{s}\left(H_{1}^{2}+H_{2}^{2}\right)
$$

Our thermometry system was not designed for the calibrated temperature measurements of the RF surface temperature; it measures the maximum temperature rise before quench with the thermometry sensor located on the outside surface in the helium bath. We show the thermometry experimental results here, to illustrate that the temperature data qualitatively agrees with RF modemixing data.

\section{EXPERIMENTAL RESULTS AND DISCUSSION}

The RF measurement results for cavities TB9NR001, TB9RI023, JLAB-LG-1 are shown in Fig 1 . TB9NR001 and TB9RI023 were produced by two industrial vendors for the ILC R\&D yield studies. Both cavities are fine grain $(\approx 50 \mu \mathrm{m})$ 9-cell ILC cavities made of high $\mathrm{RRR}(\approx 300)$ bulk niobium. JLab-LG-1 is an R\&D 9cell cavity manufactured at JLab from high RRR large grain $(\approx 5 \mathrm{~cm})$ material[25]. The standard RF qualification measurement consists of locking $\mathrm{TM}_{010} \pi$-mode resonant frequency and measuring quality factor, while step-wise ramping up the field in approximately $\mathrm{B}_{\text {peak }} \cong$ $5 \mathrm{mT}$ increments up to the quench field. TB9NR001 was limited in $\pi$ mode to $\mathrm{B}_{\text {peak }}=70 \pm 4 \mathrm{mT}$. Thermometry and OSTs measurements indicated that the cavity is limited at the equator in the fifth cell. Quenches in all odd pass-band modes were confirmed with OSTs to happen at the same location. For dual mode excitation measurements $\pi\left(f_{\mathrm{TM}_{010}}^{\pi} \cong 1.29985 \mathrm{GHz}\right)$ and $7 \pi / 9\left(f_{\mathrm{TM}_{010}}^{7 \pi / 9} \cong 1.29664 \mathrm{GHz}\right)$ modes were mixed first, later during the same cool down $3 \pi / 9\left(f_{\mathrm{TM}}^{3 \pi / 9} \cong 1.27981\right.$ $\mathrm{GHz}$ ) and $7 \pi / 9$ modes were mixed. TB9RI023 in $6 \pi / 9$, $5 \pi / 9$, and $2 \pi / 9$ modes was limited by a defect in the third cell. $6 \pi / 9\left(f_{\mathrm{TM}_{010}}^{6 \pi / 9} \cong 1.29313 \mathrm{GHz}\right)$ and $5 \pi / 9\left(f_{\mathrm{TM}_{010}}^{5 \pi / 9} \cong\right.$ $1.28908 \mathrm{GHz}$ ) were used for mode-mixing measurements, because these modes are better coupled to the cavity by the fixed coupler that we use. Following the same experimental procedure as with TB9NR001, the RF and thermometry data was recorded. In Fig. 1 the results from dual mode excitation measurements of $\pi$ \& $7 \pi / 9$ and $3 \pi / 9 \& 7 \pi / 9$ modes along with the best fits are presented. The TB9NR001 measurements best fit for $\pi$ \& $7 \pi / 9$ is $0.20 \pm 0.01$, the best fit for $3 \pi / 9 \& 7 \pi / 9$ is 0.19 \pm 0.01 . The TB9RI023 measurements best fit for $5 \pi / 9$ $\& 6 \pi / 9$ is $0.58 \pm 0.02$. JLab-LG- 1 was limited in $\pi$ mode by a defect in the sixth cell at $\mathrm{B}_{\text {peak }}=86 \pm 5 \mathrm{mT}$. The same limitation was encountered in $6 \pi / 9,4 \pi / 9$, and $\pi / 9$ modes. The results for $\pi\left(f_{\mathrm{TM}_{010}}^{\pi} \cong 1.30054 \mathrm{GHz}\right)$ and $6 \pi / 9\left(f_{\mathrm{TM}_{010}}^{\pi} \cong 1.29365 \mathrm{GHz}\right)$ mode-mixing are shown in Fig 1 . The best fit yields $0.63 \pm 0.01$.
TABLE I. In this table we summarize cavity IDs, passbands, measured end cell peak fields, and limitations for the passbands used during mode mixing measurements. Note that the listed peak magnetic field are the peak magnetic field measured in the end cell and calculated 20] peak magnetic field in the limiting cell.

\begin{tabular}{|l|c|c|c|c|}
\hline Cavity ID & mode & $\begin{array}{c}\mathrm{B}_{\text {peak }}^{\text {max }}[\mathrm{mT}] \\
\text { (end cell) }\end{array}$ & $\begin{array}{c}\mathrm{B}_{\text {peak }}^{\text {max }}[\mathrm{mT}] \\
(\text { limiting cell) }\end{array}$ & limit \\
\hline TB9NR001 & $\pi$ & 70 & 70 & $\begin{array}{c}\text { cell \#5 } \\
\text { quench }\end{array}$ \\
\hline TB9NR001 & $7 \pi / 9$ & 72 & 74 & $\begin{array}{c}\text { cell \#5 } \\
\text { quench }\end{array}$ \\
\hline TB9NR001 & $3 \pi / 9$ & 35 & 69 & $\begin{array}{c}\text { cell \#5 } \\
\text { quench }\end{array}$ \\
\hline TB9RI023 & $\pi$ & 72 & 72 & $\begin{array}{c}\text { Field } \\
\text { emission }\end{array}$ \\
\hline TB9RI023 & $6 \pi / 9$ & 149 & 146 & $\begin{array}{c}\text { cell \#3 } \\
\text { quench }\end{array}$ \\
\hline TB9RI023 & $5 \pi / 9$ & 115 & 139 & $\begin{array}{l}\text { cell \#3 } \\
\text { quench }\end{array}$ \\
\hline JLab-LG-1 & $\pi$ & 86 & 86 & $\begin{array}{l}\text { cell \#6 } \\
\text { quench }\end{array}$ \\
\hline JLab-LG-1 & $6 \pi / 9$ & 103 & 101 & $\begin{array}{l}\text { cell \#6 } \\
\text { quench }\end{array}$ \\
\hline
\end{tabular}

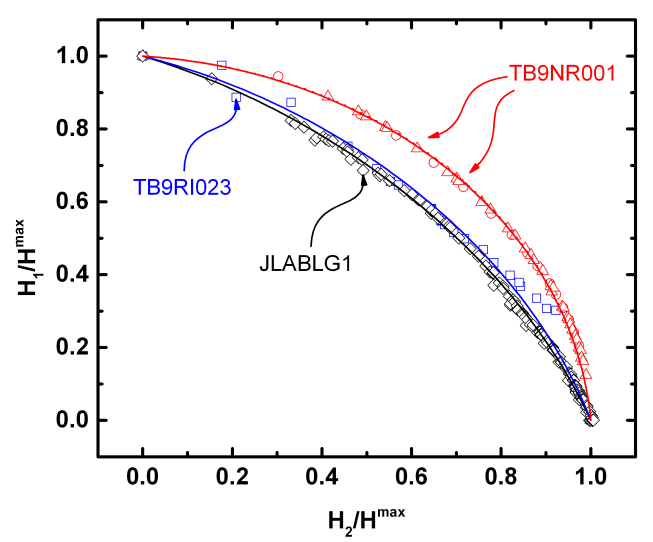

FIG. 1. RF mode-mixing measurements of TB9NR001, TB9RI023, and JLab-LG-1. Open blue squares present the data for $6 \pi / 9$ and $5 \pi / 9$ mode mixing in TB9RI023; the blue solid line present the best fit using (9) with the best fit $\alpha$ of $0.58 \pm 0.02$. Open red circles and triangles present the data for $\pi \& 7 \pi / 9$ and $3 \pi / 9 \& 7 \pi / 9$ of TB9NR001 mode mixing; the best fits are presented with solid and dot red lines; the best fits for $\alpha$ are $0.20 \pm 0.01$ and $0.19 \pm 0.01$ respectively. JLab-LG-1 $\pi$ \& $6 \pi / 9$ mode-mixing results are shown with black rhombs. The best fit $\alpha=0.63 \pm 0.00$ is shown with the solid black line.

One of the Allen-Bradley thermometers that was located at the identified quench location sites and showed the highest temperature rise before the quench was used for dual-mode thermometry measurements. The thermometer was disconnected from 2-cell thermometry DAQ multiplexer and connected to a separate 8-channel DAQ. 
The voltage across the thermometer along with the transmitted power was then recorded as a function of time for different mode-mixing amplitudes. The highest voltage before the quench for different mode-mixing amplitudes was identified from the transmitted power and the thermometer voltage trace. The voltage was then converted to the temperature rise with the calibration curve. The temperature rise data points as a function of one of the mixed fields is presented in Fig. 2 for TB9NR001 and TB9RI023. During TB9RI023 thermometry measurements we observed sharp rise in temperature after the quench for $\tilde{H}$ close to 0 or 1 , but did not see the sharp temperature rise for $\tilde{H}$ between 0.3 and 0.9 . At this time it is unclear why this changed occurred, but it was reproducible and could be a future research topic outside the scope of this paper.

As we pointed earlier, the thermometry is not calibrated against the RF surface temperature, so we did not try to fit the data with theoretical expression. Instead, we calculated the expected temperature rise for the best fit $\alpha$ from $\mathrm{RF}$ measurements. In Fig. 2 the experimental thermometry data points with the scaled overlay of fitted curves from RF data shows strong agreement between two techniques. As expected for TB9NR001 from $\mathrm{RF}$ measurement with an $\alpha \approx 0.2$ the temperature shows little variation, whereas TB9RI023 data with RF $\alpha \cong$ 0.58 shows the expected dip at about half-field value.

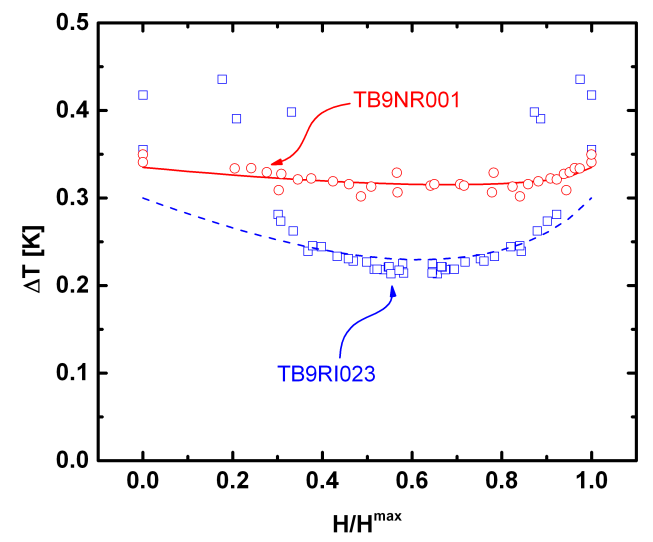

FIG. 2. Temperature rise as a function of field during modemixing measurement. Blue open squares present the data for TB9RI023; the dashed blue line is the expected dependence with $\alpha=0.58 ; \Delta \mathrm{T}_{\max }$ is set to $0.3 \mathrm{~K}$. Red open circles are the experimental measurement for TB9NR001; the solid red line is the expected dependence with $\alpha=0.2 ; \Delta \mathrm{T}_{\max }$ is set to $0.335 \mathrm{~K}$.

Using the equation (11), the quench fields from the table $T$, and the fitting parameter $\alpha$ we can infer the material critical field at the quench site for the three quenches. From the quench field in TB9NR001 of $\approx 70 \mathrm{mT}$ and $\alpha$ $=0.2$, we infer the material critical field in TB9NR001 is $\approx 350 \mathrm{mT}$. This value is about 50 percent higher than $240 \mathrm{mT}$ at $2 \mathrm{~K}$ typically assumed to be the limiting su- perheating field of niobium 3 , 26]. We speculate that the discrepancy stems from the assumption that the breakdown is magnetic, whereas in TB9NR001 the quench is likely to be a thermal quench from a normal conducting inclusion 27]. If the quench in TB9NR001 is in fact thermal then equation (6) does not describe the breakdown condition and equation (11) is not applicable. From the quench field in TB9RI023 of $\approx 140 \mathrm{mT}$ and $\alpha=0.58$, we infer the material critical field in TB9RI023 is $\approx 241$ $\mathrm{mT}$. This value compares well with $240 \mathrm{mT}$ for the generally accepted value of the superheating field. We note that in the case of TB9RI023 the quench site was off the equator, and therefore the derived quench field, which is the calculated peak magnetic field in the cell, is overestimated. From the quench field in JLAB LG-1 of $\approx 86$ $\mathrm{mT}$ and $\alpha=0.63$, we infer the material critical field in JLAB LG-1 is $\approx 137 \mathrm{mT}$. This value is about 40 percent lower than $240 \mathrm{mT}$, which can be understood, if we take into account a geometrical field enhancement factor of the large grain cavity. We note that in JLAB LG-1 a sharp feature was found at the quench location 28 .

\section{CONCLUSION}

We have studied breakdown of superconductivity in several multicell superconducting radio frequency cavities by simultaneous excitation of two $\mathrm{TM}_{010}$ pass-band modes. The experimental data is characterized by one parameter, which is related to geometry and material properties of the superconductor. Unlike measurements done in the past, which indicated a clear thermal nature of the breakdown, our measurements at higher fields present a more complex picture with interplay of both thermal and magnetic effects. The limiting defects were characterized with RF measurement and thermometry, which we have shown are independent of each other. Mode-mixing parameters calculated from RF and thermometry data show a fair agreement for both measured defects. Either of these measurement techniques, RF or thermometry, can be used to quantify the superconducting RF breakdown.

\section{ACKNOWLEDGMENTS}

We would like to thank for suggestions and useful discussions Gigi Ciovati, Rongli Geng, Charlie Reece, Bob Rimmer, and Hui Tian. We are grateful to Jefferson Lab SRF staff for helping with cavity preparation and assistance with RF tests.

This manuscript has been authored by Jefferson Science Associates, LLC under Contract No. DE-AC0506OR23177 with the U.S. Department of Energy. The United States Government retains and the publisher, by accepting the article for publication, acknowledges that the United States Government retains a non-exclusive, paid-up, irrevocable, world-wide license to publish or re- 
produce the published form of this manuscript, or allow others to do so, for United States Government purposes.
[1] R.L. Geng, G.V. Eremeev, H. Padamsee, and V.D. Shemelin. High gradient studies for ILC with singlecell re-entrant shape and elliptical shape cavities made of fine-grain and large-grain niobium. In Proceedings of the Particle Accelerator Conference, PAC'200\%, pages 23372339, June 2007.

[2] F. Furuta and K. Saito. $50 \mathrm{MV} / \mathrm{M}$ recipe for ICHIRO end groups with ethanol rinsing and wiping. In Proceedings of the $14^{\text {th }}$ International Conference on RF superconductivity, SRF'2009, pages 821-823, August 2009.

[3] R.A. French. Intrinsic Type-II superconductivity in pure niobium. Cryogenics, 8(5):301-308, 1968.

[4] F. Pei-Jen Lin and A. Gurevich. Effect of impurities on the superheating field of Type-II superconductors. Phys. Rev. B, 85:054513, Feb 2012.

[5] Mark K. Transtrum, Gianluigi Catelani, and James P. Sethna. Superheating field of superconductors within Ginzburg-Landau theory. Phys. Rev. B, 83:094505, Mar 2011.

[6] G. Catelani and James P. Sethna. Temperature dependence of the superheating field for superconductors in the high- $\kappa$ London limit. Phys. Rev. B, 78:224509, Dec 2008.

[7] T. Yogi, G. J. Dick, and J. E. Mercereau. Critical rf magnetic fields for some Type-I and Type-II superconductors. Phys. Rev. Lett., 39:826-829, Sep 1977.

[8] H. Padamsee, D. Proch, P. Kneisel, and J. Mioduszewski. Field strength limitations in superconducting cavitiesmultipacting and thermal breakdown. IEEE Transactions on Magnetics, 17(1):947 - 950, January 1981.

[9] Grigory V. Eremeev. Study of the High Field Q-Slope Using Thermometry. PhD thesis, Cornell University, 2008.

[10] Alex Gurevich. Superconducting radio-frequency fundamentals for particle accelerators. Reviews of Accelerator Science and Technology, 05:119-146, 2012.

[11] V. M. Uroev. Equations of Mathematical Physics. Yauza, Moscow, first edition, 1998. [in russian].

[12] Chien Chou, David White, and H. L. Johnston. Heat capacity in the normal and superconducting states and critical field of niobium. Phys. Rev., 109:788-796, Feb 1958.

[13] A. Gurevich. Multiscale mechanisms of SRF breakdown. Physica C: Superconductivity, 441(1-2):38 - 43, 2006. Proceedings of the 12th International Workshop on RF Superconductivity.

[14] T. Hays and H. Padamsee. Measuring the rf critical field of $\mathrm{Pb}, \mathrm{Nb}$, and $\mathrm{Nb}_{3} \mathrm{Sn}$. In Proceedings of the $8^{\text {th }}$ Workshop on RF superconductivity, SRF'1997, pages 789-794, 1997.

[15] N. Valles and M. Liepe. The superheating field of niobium: Theory and experiment. In Proceedings of the $15^{\text {th }}$ International Conference of RF Superconductivity, SRF'2011, pages 293-301, July 2011.
[16] Neil W. Ashcroft and David N. Mermin. Solid State Physics. Thomson Learning, Toronto, first edition, January 1976.

[17] R.L. Geng and A.C. Crawford. Standard procedures of ILC high gradient cavity processing and handling at Jefferson Lab. In Proceedings of the $15^{\text {th }}$ International Conference on RF superconductivity, SRF'2011, pages 391393, July 2011.

[18] G. Ciovati, T. Higo, P. Kneisel, J. Mammosser, A. Matheisen, P. Michelato, H. Padamsee, D. Reschke, T. Rothgeb, T. Saeki, K. Saito, B. Visentin, H. Weise, and Chr. Oevermann. Final surface preparation for superconducting cavities. Technical Report TTC-Report 2008-05, DESY, 2008.

[19] H. Padamsee, J. Knobloch, and T. Hays. RF Superconductivity for Accelerators. Wiley, New York, 2008.

[20] Haipeng Wang. $\mathrm{TM}_{010}$ pass band modes of TESLA 9cell cavity. Technical Report JLAB-TN-07-052, TJNAF, 2007.

[21] Z. A. Conway, D. L. Hartill, H. S. Padamsee, and E. N. Smith. Oscillating superleak transducers for quench detection in superconducting ILC cavities cooled with HeII. In Proceedings of the Linear Accelerator Conference, LINAC'2008., pages 863-865, September 2008.

[22] G. Ciovati, R. Flood, P. Kneisel, D. Machie, and M. Morrone. A 2-cell temperature mapping system for ILC cavities. Technical Report JLAB-TN-08-012, TJNAF, 2008.

[23] G. Eremeev, R. L. Geng, and A. D. Palczewski. Probing the fundamental limit of niobium in high radiofrequency fields by dual mode excitation in superconducting radiofrequency cavities. In Proceedings of the $15^{\text {th }}$ International Conference on RF superconductivity, SRF'2011, pages 746-749, July 2011.

[24] Jens Knobloch. Advanced Thermometry Studies of Superconducting RF Cavities. PhD thesis, Cornell University, 1997.

[25] P. Kneisel, G. Ciovati, and A. Crawford. Status of inhouse 9-cell fabrication and testing at JLab. Technical Report JLAB-TN-08-083, TJNAF, 2008.

[26] J. Matricon and D. Saint-James. Superheating fields in superconductors. Physics Letters A, 24(5):241 - 242, 1967.

[27] A.D. Palczewski, G. Eremeev, and R.L. Geng. Design, construction, and initial test of high spatial resolution thermometry arrays for detection of surface temperature profiles on SRF cavities in superfluid helium. In Proceedings of the $15^{\text {th }}$ International Conference on RF superconductivity, SRF'2011, pages 755-758, July 2011.

[28] K. Watanabe, H. Hayano, and Y. Iwashita. Cavity inspection and repair techniques. In Proceedings of the $15^{\text {th }}$ International Conference on RF superconductivity, SRF'2011, pages 598-602, July 2011. 